Acta Crystallographica Section E

Structure Reports

Online

ISSN 1600-5368

\section{2-(3-Methyl-2-nitrophenyl)-4,5-dihydro- 1,3-oxazole}

\section{Dongwei Lei, ${ }^{a}$ Huibin Yang, ${ }^{b}$ Bin $\mathrm{Li}^{\mathrm{b}}$ and Zhuo Kang ${ }^{\mathrm{b} *}$}

aShenyang Institute of Chemical Technology, Shenyang 110142, People's Republic of China, and ${ }^{\mathbf{b}}$ Agrochemicals Division, Shenyang Research Institute of Chemical Industry, Shenyang 110021, People's Republic of China

Correspondence e-mail: kangzhuo@sinochem.com

Received 27 November 2008; accepted 4 December 2008

Key indicators: single-crystal X-ray study; $T=296 \mathrm{~K}$; mean $\sigma(\mathrm{C}-\mathrm{C})=0.003 \AA$; $R$ factor $=0.037 ; w R$ factor $=0.110 ;$ data-to-parameter ratio $=10.4$.

In the title compound, $\mathrm{C}_{10} \mathrm{H}_{10} \mathrm{~N}_{2} \mathrm{O}_{3}$, an intermediate in the synthesis of anthranilamide insecticides, all the non- $\mathrm{H}$ atoms except the nitro-group $\mathrm{O}$ atom lie on a crystallographic mirror plane. The $\mathrm{H}$ atoms of the methyl group are disordered over two sets of sites with equal occupancies. In the crystal structure, $\mathrm{C}-\mathrm{H} \cdots \mathrm{N}$ links lead to chains of molecules propagating in [100].

\section{Related literature}

For background to anthranilamide compounds, a new class of inseticides, see: Lahm et al. (2003, 2005).<smiles>Cc1cccc(C2=NCCO2)c1[N+](=O)[O-]</smiles>

\section{Experimental}

Crystal data

$\mathrm{C}_{10} \mathrm{H}_{10} \mathrm{~N}_{2} \mathrm{O}_{3}$

$M_{r}=206.20$
Monoclinic, $P 2_{1} / m$

$a=7.7767(10) \AA$

$b=7.3370(10) \AA$

$c=8.6468(12) \AA$

$\beta=99.414$ (2)

$V=486.72(11) \AA^{3}$

$Z=2$

Mo $K \alpha$ radiation

$\mu=0.11 \mathrm{~mm}^{-1}$

$T=296(2) \mathrm{K}$

$0.24 \times 0.22 \times 0.18 \mathrm{~mm}$

Data collection

Bruker SMART CCD

diffractometer

Absorption correction: multi-scan

(SADABS; Bruker, 2001)

$T_{\min }=0.834, T_{\max }=1.000$

(expected range $=0.818-0.981)$

Refinement

$R\left[F^{2}>2 \sigma\left(F^{2}\right)\right]=0.037$

$w R\left(F^{2}\right)=0.110$

$S=1.07$

937 reflections

2462 measured reflections 937 independent reflections 842 reflections with $I>2 \sigma(I)$ $R_{\text {int }}=0.011$

Table 1

Hydrogen-bond geometry $\left(\AA{ }^{\circ}\right)$.

\begin{tabular}{lllll}
\hline$D-\mathrm{H} \cdots A$ & $D-\mathrm{H}$ & $\mathrm{H} \cdots A$ & $D \cdots A$ & $D-\mathrm{H} \cdots A$ \\
\hline $\mathrm{C} 4-\mathrm{H} 4 \cdots \mathrm{N} 1^{\mathrm{i}}$ & 0.93 & 2.60 & $3.508(3)$ & 167
\end{tabular}

Symmetry code: (i) $x-1, y, z$.

Data collection: SMART (Bruker, 2005); cell refinement: SAINT (Bruker, 2005); data reduction: $S A I N T$; $\operatorname{program}(\mathrm{s})$ used to solve structure: SHELXS97 (Sheldrick, 2008); program(s) used to refine structure: SHELXL97 (Sheldrick, 2008); molecular graphics: SHELXTL (Sheldrick, 2008); software used to prepare material for publication: SHELXTL.

Supplementary data and figures for this paper are available from the IUCr electronic archives (Reference: HB2868).

\section{References}

Bruker (2005). SMART and SAINT. Bruker AXS Inc., Madison, Wisconsin, USA.

Lahm, G. P., Selby, T. P., Freudenberger, J. H., Stevenson, T. M., Myers, B. J., Seburyamo, G., Smith, B. K., Flexner, L., Clark, C. E. \& Cordova, D. (2005) Bioorg. Med. Chem. Lett. 15, 4898-4906.

Lahm, G. P., Selby, T. P. \& Stevenson, T. M. (2003). International Patent WO 03/015 519.

Sheldrick, G. M. (2008). Acta Cryst. A64, 112-122. 


\section{supporting information}

Acta Cryst. (2009). E65, o54 [doi:10.1107/S1600536808040920]

\section{2-(3-Methyl-2-nitrophenyl)-4,5-dihydro-1,3-oxazole}

\section{Dongwei Lei, Huibin Yang, Bin Li and Zhuo Kang}

\section{S1. Comment}

Anthranilamide compounds as a new class of inseticides are characterized by their high levels of insecticidal activity, nocross resistance to existing insecticides, safety to off-target animal and low toxicity to mammals (Lahm et al. 2003, 2005)

The title compound (I) as an intermediate for preparing Chlorantraniliprole analogs plays an important role in identifying the configuration of two possible products.

In the molecular structure of (I), (Fig. 1) all the non-hydrogen atoms except the nitro-group $\mathrm{O}$ atom lie on a crystallographic mirror plane. In the crystal, $\mathrm{C}-\mathrm{H} \cdots \mathrm{N}$ links lead to chains of molecules propagating in [100].

\section{S2. Experimental}

2-Bromoethanamine hydrobromide $(10.25 \mathrm{~g}, 50 \mathrm{mmol})$ and 3-methyl-2-nitrobenzoyl chloride $(9.98 \mathrm{~g}, 50 \mathrm{mmol})$ were added into dichloromethane $(200 \mathrm{ml})$, then triethylamine $(16.70 \mathrm{~g}, 165 \mathrm{mmol})$ was added. The mixture was heated to reflux for $14 \mathrm{~h}$ and cooled down to room temperature, washed with water and brine, dried by anhydrous sulfate magnesium, then evaporated to give the title compound as a white solid. The product was dissolved in dichloromethane and left to stand at room temperature and colourless blocks of (I) were obtained.

Anal. Calcd for $\mathrm{C}_{10} \mathrm{H}_{10} \mathrm{~N}_{2} \mathrm{O}_{3}$ : C, 58.25; H, 4.89; N, 13.59; O, 23.28. Found: C, 58.20; H, 4.90; N, 13.61; O, $23.25{ }^{1} \mathrm{H}$ $\operatorname{NMR}\left(\mathrm{CDCl}_{3}\right): 2.35\left(\mathrm{~s}, 3 \mathrm{H}, \mathrm{CH}_{3}\right), 4.06\left(\mathrm{t}, \mathrm{J}=9.8 \mathrm{~Hz}, 2 \mathrm{H}, \mathrm{CH}_{2}\right), 4.40\left(\mathrm{t}, \mathrm{J}=9.6 \mathrm{~Hz}, 2 \mathrm{H}, \mathrm{CH}_{2}\right), 7.41-7.43(\mathrm{~m}, 2 \mathrm{H}), 7.78-7.81$ $(\mathrm{m}, 1 \mathrm{H})$.

\section{S3. Refinement}

Although all $\mathrm{H}$ atoms were visible in difference maps, they were finally placed in geometrically calculated positions, with $\mathrm{C}-\mathrm{H}$ distances in the range $0.93-0.96 \AA$, and included in the final refinement in the riding model approximation, with $U_{\text {iso }}(\mathrm{H})=1.2 U_{\text {eq }}(\mathrm{C})$ for aromatic and $U_{\text {iso }}(\mathrm{H})=1.5 U_{\text {eq }}(\mathrm{C})$ for methyl $\mathrm{H}$ atoms. 


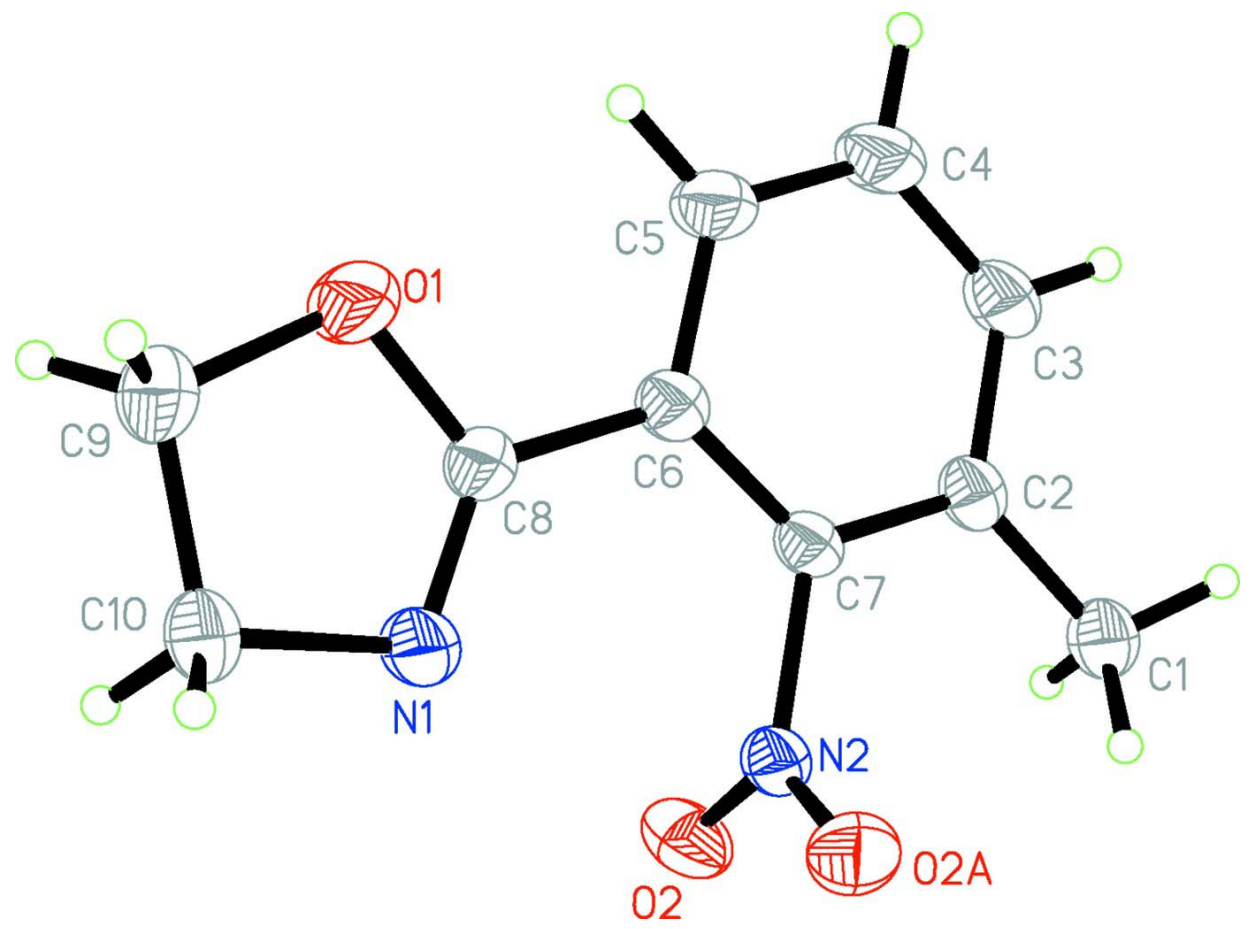

\section{Figure 1}

The molecular structure of (I), with $30 \%$ probability displacement ellipsoids for the non-hydrogen atoms. Symmetry code: A x, 1/2-y, z.

\section{2-(3-Methyl-2-nitrophenyl)-4,5-dihydro-1,3-oxazole}

\section{Crystal data}

$\mathrm{C}_{10} \mathrm{H}_{10} \mathrm{~N}_{2} \mathrm{O}_{3}$

$M_{r}=206.20$

Monoclinic, $P 2_{1} / m$

$a=7.7767(10) \AA$

$b=7.337(1) \AA$

$c=8.6468(12) \AA$

$\beta=99.414(2)^{\circ}$

$V=486.72(11) \AA^{3}$

$Z=2$

\section{Data collection}

Bruker SMART CCD

diffractometer

Radiation source: fine-focus sealed tube

Graphite monochromator

$\omega$ scans

Absorption correction: multi-scan

(SADABS; Bruker, 2001)

$T_{\min }=0.834, T_{\max }=1.000$
$F(000)=216$

$D_{\mathrm{x}}=1.407 \mathrm{Mg} \mathrm{m}^{-3}$

Mo $K \alpha$ radiation, $\lambda=0.71073 \AA$

Cell parameters from 1713 reflections

$\theta=2.7-27.9^{\circ}$

$\mu=0.11 \mathrm{~mm}^{-1}$

$T=296 \mathrm{~K}$

BLOCK, colourless

$0.24 \times 0.22 \times 0.18 \mathrm{~mm}$

2462 measured reflections

937 independent reflections

842 reflections with $I>2 \sigma(I)$

$R_{\text {int }}=0.011$

$\theta_{\max }=25.0^{\circ}, \theta_{\min }=2.4^{\circ}$

$h=-9 \rightarrow 9$

$k=-6 \rightarrow 8$

$l=-10 \rightarrow 9$ 


\section{Refinement}

Refinement on $F^{2}$

Least-squares matrix: full

$R\left[F^{2}>2 \sigma\left(F^{2}\right)\right]=0.037$

$w R\left(F^{2}\right)=0.110$

$S=1.07$

937 reflections

90 parameters

0 restraints

Primary atom site location: structure-invariant direct methods
Secondary atom site location: difference Fourier map

Hydrogen site location: inferred from neighbouring sites

$\mathrm{H}$-atom parameters constrained

$w=1 /\left[\sigma^{2}\left(F_{\mathrm{o}}^{2}\right)+(0.0583 P)^{2}+0.1052 P\right]$ where $P=\left(F_{\mathrm{o}}{ }^{2}+2 F_{\mathrm{c}}{ }^{2}\right) / 3$

$(\Delta / \sigma)_{\max }<0.001$

$\Delta \rho_{\max }=0.17 \mathrm{e} \AA^{-3}$

$\Delta \rho_{\min }=-0.15$ e $\AA^{-3}$

Special details

Geometry. All e.s.d.'s (except the e.s.d. in the dihedral angle between two l.s. planes) are estimated using the full covariance matrix. The cell e.s.d.'s are taken into account individually in the estimation of e.s.d.'s in distances, angles and torsion angles; correlations between e.s.d.'s in cell parameters are only used when they are defined by crystal symmetry. An approximate (isotropic) treatment of cell e.s.d.'s is used for estimating e.s.d.'s involving 1.s. planes.

Refinement. Refinement of $F^{2}$ against ALL reflections. The weighted $R$-factor $w R$ and goodness of fit $S$ are based on $F^{2}$, conventional $R$-factors $R$ are based on $F$, with $F$ set to zero for negative $F^{2}$. The threshold expression of $F^{2}>\sigma\left(F^{2}\right)$ is used only for calculating $R$-factors(gt) etc. and is not relevant to the choice of reflections for refinement. $R$-factors based on $F^{2}$ are statistically about twice as large as those based on $F$, and $R$ - factors based on ALL data will be even larger.

Fractional atomic coordinates and isotropic or equivalent isotropic displacement parameters $\left(\AA^{2}\right)$

\begin{tabular}{llllll}
\hline & $x$ & $y$ & $z$ & $U_{\text {iso }} / U_{\text {eq }}$ & Occ. $(<1)$ \\
\hline O1 & $0.2190(2)$ & 0.2500 & $1.12373(17)$ & $0.0794(6)$ & \\
O2 & $0.33918(12)$ & $0.10351(17)$ & $0.61731(13)$ & $0.0628(4)$ & \\
N1 & $0.4057(2)$ & 0.2500 & $0.95534(19)$ & $0.0617(6)$ & \\
N2 & $0.27535(19)$ & 0.2500 & $0.64083(17)$ & $0.0428(4)$ & \\
C1 & $-0.0249(3)$ & 0.2500 & $0.4054(2)$ & $0.0520(5)$ & \\
H1A & 0.0705 & 0.3268 & 0.3893 & $0.078^{*}$ & 0.50 \\
H1B & -0.1309 & 0.2952 & 0.3449 & $0.078^{*}$ & 0.50 \\
H1C & -0.0041 & 0.1280 & 0.3728 & $0.078^{*}$ & 0.50 \\
C2 & $-0.0410(2)$ & 0.2500 & $0.5759(2)$ & $0.0430(5)$ & \\
C3 & $-0.2030(3)$ & 0.2500 & $0.6245(3)$ & $0.0533(5)$ & \\
H3 & -0.3034 & 0.2500 & 0.5495 & $0.064^{*}$ & \\
C4 & $-0.2181(3)$ & 0.2500 & $0.7801(3)$ & $0.0630(6)$ & \\
H4 & -0.3281 & 0.2500 & 0.8092 & $0.076^{*}$ & \\
C5 & $-0.0709(3)$ & 0.2500 & $0.8946(3)$ & $0.0589(6)$ & \\
H5 & -0.0828 & 0.2500 & 0.9998 & $0.071^{*}$ & \\
C6 & $0.0946(2)$ & 0.2500 & $0.8531(2)$ & $0.0434(5)$ & \\
C7 & $0.1040(2)$ & 0.2500 & $0.6938(2)$ & $0.0381(4)$ & \\
C8 & $0.2508(2)$ & 0.2500 & $0.9762(2)$ & $0.0428(5)$ & \\
C9 & $0.3878(3)$ & 0.2500 & $1.2242(3)$ & $0.0671(7)$ & \\
H9 & 0.4021 & 0.1424 & 1.2902 & $0.081^{*}$ & \\
C10 & $0.5156(3)$ & 0.2500 & $1.1111(2)$ & $0.0580(6)$ & \\
H10 & 0.5890 & 0.3576 & 1.1251 & $0.070^{*}$ & \\
& & & &
\end{tabular}


Atomic displacement parameters $\left(\AA^{2}\right)$

\begin{tabular}{lllllll}
\hline & $U^{11}$ & $U^{22}$ & $U^{33}$ & $U^{12}$ & $U^{13}$ & $U^{23}$ \\
\hline O1 & $0.0487(9)$ & $0.1510(17)$ & $0.0384(8)$ & 0.000 & $0.0066(7)$ & 0.000 \\
O2 & $0.0475(6)$ & $0.0714(8)$ & $0.0701(8)$ & $0.0137(5)$ & $0.0117(5)$ & $-0.0128(5)$ \\
N1 & $0.0353(9)$ & $0.1100(16)$ & $0.0376(9)$ & 0.000 & $-0.0005(7)$ & 0.000 \\
N2 & $0.0323(8)$ & $0.0582(10)$ & $0.0363(8)$ & 0.000 & $0.0013(6)$ & 0.000 \\
C1 & $0.0451(11)$ & $0.0637(13)$ & $0.0437(11)$ & 0.000 & $-0.0033(8)$ & 0.000 \\
C2 & $0.0358(10)$ & $0.0443(10)$ & $0.0463(10)$ & 0.000 & $-0.0010(8)$ & 0.000 \\
C3 & $0.0316(9)$ & $0.0658(13)$ & $0.0594(13)$ & 0.000 & $-0.0020(8)$ & 0.000 \\
C4 & $0.0322(10)$ & $0.0932(17)$ & $0.0649(14)$ & 0.000 & $0.0114(9)$ & 0.000 \\
C5 & $0.0397(11)$ & $0.0885(16)$ & $0.0505(12)$ & 0.000 & $0.0130(9)$ & 0.000 \\
C6 & $0.0347(10)$ & $0.0516(11)$ & $0.0434(10)$ & 0.000 & $0.0046(8)$ & 0.000 \\
C7 & $0.0290(8)$ & $0.0422(10)$ & $0.0428(10)$ & 0.000 & $0.0047(7)$ & 0.000 \\
C8 & $0.0408(10)$ & $0.0521(11)$ & $0.0352(9)$ & 0.000 & $0.0057(7)$ & 0.000 \\
C9 & $0.0550(13)$ & $0.1013(19)$ & $0.0413(11)$ & 0.000 & $-0.0030(10)$ & 0.000 \\
C10 & $0.0445(11)$ & $0.0834(16)$ & $0.0418(11)$ & 0.000 & $-0.0058(8)$ & 0.000 \\
\hline
\end{tabular}

Geometric parameters $\left(\AA,{ }^{\circ}\right)$

\begin{tabular}{llll}
\hline $\mathrm{O} 1-\mathrm{C} 8$ & $1.339(2)$ & $\mathrm{C} 3-\mathrm{C} 4$ & $1.370(3)$ \\
$\mathrm{O} 1-\mathrm{C} 9$ & $1.451(3)$ & $\mathrm{C} 3-\mathrm{H} 3$ & 0.9300 \\
$\mathrm{O} 2-\mathrm{N} 2$ & $1.2149(13)$ & $\mathrm{C} 4-\mathrm{C} 5$ & $1.385(3)$ \\
$\mathrm{N} 1-\mathrm{C} 8$ & $1.247(2)$ & $\mathrm{C} 4-\mathrm{H} 4$ & 0.9300 \\
$\mathrm{~N} 1-\mathrm{C} 10$ & $1.472(2)$ & $\mathrm{C} 5-\mathrm{C} 6$ & $1.391(3)$ \\
$\mathrm{N} 2-\mathrm{O} 2$ & $\mathrm{C} 5-\mathrm{H} 5$ & 0.9300 \\
$\mathrm{~N} 2-\mathrm{C} 7$ & $1.2149(13)$ & $\mathrm{C} 6-\mathrm{C} 7$ & $1.391(3)$ \\
$\mathrm{C} 1-\mathrm{C} 2$ & $1.478(2)$ & $\mathrm{C} 6-\mathrm{C} 8$ & $1.478(3)$ \\
$\mathrm{C} 1-\mathrm{H} 1 \mathrm{~A}$ & $1.501(3)$ & $\mathrm{C} 9-\mathrm{C} 10$ & $1.504(3)$ \\
$\mathrm{C} 1-\mathrm{H} 1 \mathrm{~B}$ & 0.9600 & $\mathrm{C} 9-\mathrm{H} 9$ & 0.9700 \\
$\mathrm{C} 1-\mathrm{H} 1 \mathrm{C}$ & 0.9600 & $\mathrm{C} 9-\mathrm{H} 9^{\mathrm{i}}$ & 0.9700 \\
$\mathrm{C} 2-\mathrm{C} 7$ & 0.9600 & $\mathrm{C} 10-\mathrm{H} 10$ & 0.9700 \\
$\mathrm{C} 2-\mathrm{C} 3$ & $1.391(2)$ & $\mathrm{C} 10-\mathrm{H} 10^{\mathrm{i}}$ & 0.9700 \\
& $1.391(3)$ & $\mathrm{C} 6-\mathrm{C} 5-\mathrm{H} 5$ & 119.8 \\
$\mathrm{C} 8-\mathrm{O} 1-\mathrm{C} 9$ & $\mathrm{C} 7-\mathrm{C} 6-\mathrm{C} 5$ & $117.07(18)$ \\
$\mathrm{C} 8-\mathrm{N} 1-\mathrm{C} 10$ & $106.31(16)$ & $\mathrm{C} 7-\mathrm{C} 6-\mathrm{C} 8$ & $122.89(17)$ \\
$\mathrm{O} 2-\mathrm{N} 2-\mathrm{O} 2$ & $107.34(17)$ & $\mathrm{C} 5-\mathrm{C} 6-\mathrm{C} 8$ & $120.04(18)$ \\
$\mathrm{O} 2-\mathrm{N} 2-\mathrm{C} 7$ & $124.43(16)$ & $\mathrm{C} 2-\mathrm{C} 7-\mathrm{C} 6$ & $123.93(17)$ \\
$\mathrm{O} 2-\mathrm{N} 2-\mathrm{C} 7$ & $117.75(8)$ & $\mathrm{C} 6-\mathrm{C} 7-\mathrm{N} 2$ & $115.90(16)$ \\
$\mathrm{C} 2-\mathrm{C} 1-\mathrm{H} 1 \mathrm{~A}$ & $117.75(8)$ & $\mathrm{N} 1-\mathrm{C} 8-\mathrm{O} 1$ & $120.18(15)$ \\
$\mathrm{C} 2-\mathrm{C} 1-\mathrm{H} 1 \mathrm{~B}$ & 109.5 & $\mathrm{~N} 1-\mathrm{C} 8-\mathrm{C} 6$ & $118.10(17)$ \\
$\mathrm{H} 1 \mathrm{~A}-\mathrm{C} 1-\mathrm{H} 1 \mathrm{~B}$ & 109.5 & $\mathrm{O} 1-\mathrm{C} 8-\mathrm{C} 6$ & $126.55(17)$ \\
$\mathrm{C} 2-\mathrm{C} 1-\mathrm{H} 1 \mathrm{C}$ & 109.5 & $\mathrm{O} 1-\mathrm{C} 9-\mathrm{C} 10$ & $115.35(16)$ \\
$\mathrm{H} 1 \mathrm{~A}-\mathrm{C} 1-\mathrm{H} 1 \mathrm{C}$ & 109.5 & $\mathrm{C} 10-\mathrm{C} 9-\mathrm{H} 99^{\mathrm{i}}$ & $103.87(16)$ \\
$\mathrm{H} 1 \mathrm{~B}-\mathrm{C} 1-\mathrm{H} 1 \mathrm{C}$ & 109.5 & & 111.0 \\
$\mathrm{C} 7-\mathrm{C} 2-\mathrm{C} 3$ & 109.5 & $116.38(18)$ & 111.0 \\
$\mathrm{C} 7-\mathrm{C} 2-\mathrm{C} 1$ & $121.46(17)$ & $\mathrm{O} 9$ & \\
$\mathrm{C} 3-\mathrm{C} 2-\mathrm{C} 1$ & & &
\end{tabular}




$\begin{array}{llll}\mathrm{C} 4-\mathrm{C} 3-\mathrm{C} 2 & 121.60(18) & \mathrm{C} 10-\mathrm{C} 9-\mathrm{H} 9^{\mathrm{i}} & 111.0 \\ \mathrm{C} 4-\mathrm{C} 3-\mathrm{H} 3 & 119.2 & \mathrm{H} 9-\mathrm{C} 9-\mathrm{H} 9^{\mathrm{i}} & 109.0 \\ \mathrm{C} 2-\mathrm{C} 3-\mathrm{H} 3 & 119.2 & \mathrm{~N} 1-\mathrm{C} 10-\mathrm{C} 9 & 104.38(16) \\ \mathrm{C} 3-\mathrm{C} 4-\mathrm{C} 5 & 120.52(19) & \mathrm{N} 1-\mathrm{C} 10-\mathrm{H} 10 & 110.9 \\ \mathrm{C} 3-\mathrm{C} 4-\mathrm{H} 4 & 119.7 & \mathrm{C} 9-\mathrm{C} 10-\mathrm{H} 10 & 110.9 \\ \mathrm{C} 5-\mathrm{C} 4-\mathrm{H} 4 & 119.7 & \mathrm{~N} 1-\mathrm{C} 10-\mathrm{H} 10^{\mathrm{i}} & 110.9 \\ \mathrm{C} 4-\mathrm{C} 5-\mathrm{C} 6 & 120.5(2) & \mathrm{C} 9-\mathrm{C} 10-\mathrm{H} 10^{\mathrm{i}} & 108.9 \\ \mathrm{C} 4-\mathrm{C} 5-\mathrm{H} 5 & 119.8 & \mathrm{H} 10-\mathrm{C} 10-\mathrm{H} 10^{\mathrm{i}} & -88.46(13) \\ & & & -91.54(13) \\ \mathrm{C} 7-\mathrm{C} 2-\mathrm{C} 3-\mathrm{C} 4 & 0.0 & \mathrm{O} 2-\mathrm{N} 2-\mathrm{C} 7-\mathrm{C} 2 & 91.54(13) \\ \mathrm{C} 1-\mathrm{C} 2-\mathrm{C} 3-\mathrm{C} 4 & 180.0 & \mathrm{O} 2-\mathrm{N} 2-\mathrm{C} 7-\mathrm{C} 6 & 0.0 \\ \mathrm{C} 2-\mathrm{C} 3-\mathrm{C} 4-\mathrm{C} 5 & 0.0 & \mathrm{O} 2-\mathrm{N} 2-\mathrm{C} 7-\mathrm{C} 6 & 180.0 \\ \mathrm{C} 3-\mathrm{C} 4-\mathrm{C} 5-\mathrm{C} 6 & 0.0 & \mathrm{C} 10-\mathrm{N} 1-\mathrm{C} 8-\mathrm{O} 1 & 0.0 \\ \mathrm{C} 4-\mathrm{C} 5-\mathrm{C} 6-\mathrm{C} 7 & 0.0 & \mathrm{C} 10-\mathrm{N} 1-\mathrm{C} 8-\mathrm{C} 6 & 180.0 \\ \mathrm{C} 4-\mathrm{C} 5-\mathrm{C} 6-\mathrm{C} 8 & 180.0 & \mathrm{C} 9-\mathrm{O} 1-\mathrm{C} 8-\mathrm{N} 1 & 0.0 \\ \mathrm{C} 3-\mathrm{C} 2-\mathrm{C} 7-\mathrm{C} 6 & 0.0 & \mathrm{C} 9-\mathrm{O} 1-\mathrm{C} 8-\mathrm{C} 6 & 180.0 \\ \mathrm{C} 1-\mathrm{C} 2-\mathrm{C} 7-\mathrm{C} 6 & 180.0 & \mathrm{C} 7-\mathrm{C} 6-\mathrm{C} 8-\mathrm{N} 1 & 180.0 \\ \mathrm{C} 3-\mathrm{C} 2-\mathrm{C} 7-\mathrm{N} 2 & 180.0 & \mathrm{C} 5-\mathrm{C} 6-\mathrm{C} 8-\mathrm{N} 1 & 0.0 \\ \mathrm{C} 1-\mathrm{C} 2-\mathrm{C} 7-\mathrm{N} 2 & 0.0 & \mathrm{C} 7-\mathrm{C} 6-\mathrm{C} 8-\mathrm{O} 1 & 0.0 \\ \mathrm{C} 5-\mathrm{C} 6-\mathrm{C} 7-\mathrm{C} 2 & 0.0 & \mathrm{C} 5-\mathrm{C} 6-\mathrm{C} 8-\mathrm{O} 1 & 0.0 \\ \mathrm{C} 8-\mathrm{C} 6-\mathrm{C} 7-\mathrm{C} 2 & 180.0 & \mathrm{C} 8-\mathrm{O} 1-\mathrm{C} 9-\mathrm{C} 10 & 0.0 \\ \mathrm{C} 5-\mathrm{C} 6-\mathrm{C} 7-\mathrm{N} 2 & 180.0 & \mathrm{C} 8-\mathrm{N} 1-\mathrm{C} 10-\mathrm{C} 9 & \\ \mathrm{C} 8-\mathrm{C} 6-\mathrm{C} 7-\mathrm{N} 2 & 0.0 & \mathrm{C} 9-\mathrm{C} 10-\mathrm{N} 1 & \\ \mathrm{O} 2-\mathrm{N} 2-\mathrm{C} 7-\mathrm{C} 2 & 88.46(13) & & \end{array}$

Symmetry code: (i) $x,-y+1 / 2, z$.

Hydrogen-bond geometry $\left(\AA,{ }^{\circ}\right)$

\begin{tabular}{lllll}
\hline$D-\mathrm{H} \cdots A$ & $D-\mathrm{H}$ & $\mathrm{H} \cdots A$ & $D \cdots A$ & $D-\mathrm{H} \cdots A$ \\
\hline $\mathrm{C} 4-\mathrm{H} 4 \cdots \mathrm{N} 1^{\mathrm{ii}}$ & 0.93 & 2.60 & $3.508(3)$ & 167 \\
\hline
\end{tabular}

Symmetry code: (ii) $x-1, y, z$. 